\title{
INVESTIGACIONES
}

\section{Tecnología robótica en contextos escolares vulnerables con estudiantes de la etnia Mapuche*}

\author{
Robotics technology in deprived school contexts with Mapuche students \\ Tecnologia robótica em contextos vulneráveis com estudantes da etnia Mapuche
}

\author{
Pedro Hepp K., ${ }^{a}$ María Eugenia Merino D., ${ }^{b}$ María Victoria Barriga J., ${ }^{c}$ Andrea Huircapán A. ${ }^{d}$ \\ âPontificia Universidad Católica de Chile, Facultad de Educación. Telf.: 56-2-23545350. Correo electrónico: phepp@uc.cl \\ ${ }^{b}$ Universidad Católica de Temuco, Facultad de Educación. Telf.: 56-2-205314. Correo electrónico: mmerino@uct.cl \\ ${ }^{c}$ Universidad Católica de Temuco, Facultad de Artes y Humanidades- Telf.: 56-2-55-3797. Correo electrónico: vbarriga@uct.cl \\ ${ }^{\mathrm{d}}$ TIDE SA. Telf.: 56-2-742242. Correo electrónico: andreahuircapan@ gmail.com
}

\begin{abstract}
RESUMEN
Este artículo presenta los principales aspectos de una investigación cuyo objetivo fue probar una propuesta educativa intercultural utilizando robots con profesores y alumnos de la etnia Mapuche de Educación Parvularia, de Primero y Segundo año Básico. Todos los componentes (robots, guías de trabajo, materiales para aula) fueron construidos en el marco de la investigación y utilizados en escuelas de la Región de La Araucanía con población escolar mapuche de alta vulnerabilidad. Los resultados muestran que este tipo de recursos tecnológicos tiene gran aceptación en los escolares y que los profesores son capaces de integrarlos en sus didácticas. Sin embargo, también se concluye que los profesores requieren de un período de capacitación pedagógica relacionado con el currículum y acompañamiento en aula, para integrarlos efectivamente a sus prácticas.
\end{abstract}

Palabras Clave: robótica, interculturalidad, educación

\begin{abstract}
This article presents the main aspects of a research whose goal was to evaluate an educational proposal using robots with teachers and Mapuche students in pre- $\mathrm{K}$ to 2nd grade. Every component (robots, learning materials) was designed and built during the research and used in schools of the Araucania region with highly vulnerable Mapuche students. Results show that resources of this kind have are appealed by students, and teachers are able to integrate them in their didactics. However, teachers require pedagogical training and classroom assistance in order to effectively integrate them into their praxis.
\end{abstract}

Keywords: robotics, multiculturalism, education.

\footnotetext{
* Este trabajo es producto del proyecto Fondef TIC-EDU TE08I1013 "Diseño, desarrollo y validación de material educativo con soporte robótico para contextos interculturales". Se agradece a Conicyt el apoyo prestado a esta investigación.
} 


\section{RESUMO}

Este artigo apresenta os principais aspectos de uma investigação cujo objectivo foi provar uma proposta educativa intercultural utilizando robôs com professores e alunos da etnia Mapuche da educação pré-escolar, do primeiro e segundo ano no ensino fundamental. Todos os componentes (robôs, guias de trabalho, materiais para sala) foram construídos no marco da investigação e utilizados em escolas da região da Araucanía, com população Mapuche de alta vulnerabilidade. Os resultados mostram que este tipo de recursos tecnológicos têm grande aceitação nos alunos e que os professores são capazes de integra-los nas suas didáticas. No entanto, também se conclui que os professores requerem de um período de capacitação pedagógica relacionado com o currículo e acompanhamento na sala de aula, para os integrar efetivamente nas suas práticas.

Palavras chave: robótica, multiculturalismo, educação.

\section{INTRODUCCIÓN}

\subsection{EDUCACIÓN INTERCULTURAL}

La población en Chile está caracterizada por tener influencias de diversos grupos étnicos originarios, los cuales ofrecen desafíos educativos especiales al integrarse los niños de estas etnias al sistema escolar chileno. Teniendo en cuenta esta pluralidad, en la década de 1990 se inician en el país reformas en las políticas educacionales que incluyen el desarrollo de una educación intercultural que propenda a la transformación de las relaciones entre culturas, grupos humanos y lenguas desde una perspectiva de equidad, de pertinencia y de relevancia curricular. Es así como las actuales Bases Curriculares de la Educación Parvularia en el marco de sus orientaciones valóricas, señalan que dentro de los ambientes culturales en los que interactúa el niño y la niña

"son fundamentales aquellos que corresponden a su cultura de pertenencia, ya que contribuyen significativamente a la formación de su identidad, autoestima y sentidos más profundos. El respeto y la valorización de la diversidad étnica, lingüística y cultural de las diversas comunidades del país hacen necesario su reconocimiento e incorporación en la construcción e implementación curricular" (MINEDUC, 2005:5)

La educación intercultural hace referencia a una tendencia reformadora en la práctica educativa, con la que se intenta responder a la diversidad provocada por la confrontación y convivencia de diferentes grupos étnicos y culturales dentro de una sociedad (Merino et al., 2008). Pese a los esfuerzos por mejorar los resultados de aprendizaje en todo el país, la mayoría de las escuelas rurales y urbano-marginales de la Región de la Araucanía presentan magros resultados en el logro de competencias básicas para su formación, tal como lo demuestran los resultados de la prueba SIMCE de $4^{\circ}$ Año Básico (SIMCE, 2012). Estos resultados se deberían, en parte, a que los aprendizajes no han sido significativos para los alumnos; a que esta prueba no considera que los estudiantes pertenecen a una etnia diferente; y a la dificultad adicional que significa para los niños asumir su condición étnica en tanto miembros de una minoría indígena percibida como un grupo de bajo prestigio por la sociedad mayoritaria, que además es objeto frecuente de prejuicio y discriminación por parte de la sociedad no mapuche (Merino et al., 2009, 2008). 
Nuestra propuesta postula que el modo de superar los problemas que se derivan de las relaciones interculturales asimétricas es mediante el fomento de la comunicación intercultural en la escuela, entendida ésta como "el proceso de intercambio simbólico mediante el cual individuos de dos (o más) comunidades culturales diferentes negocian significados compartidos en una situación interactiva" (Ting-Toomey, 1999). El concepto clave que subyace es la interculturalidad que describe relaciones y encuentros entre grupos humanos que poseen diferentes maneras de pensar, sentir y actuar, y se sustenta en la necesidad de que los grupos culturalmente distintos establezcan relaciones basadas en el respeto y desde planos de igualdad (Schmelkes, 2006). Para Ting-Toomey (1999), la comunicación intercultural se compone de cuatro dimensiones: el intercambio simbólico referido al uso de símbolos verbales o no verbales por parte de uno o más individuos para lograr significados compartidos; la naturaleza transaccional de la comunicación intercultural por cuanto todo discurso se basa en el intercambio de significados, los que una vez pronunciados se tornan irreversibles desde una perspectiva comunicativa por cuanto todo acto de lenguaje es acción desplegada hacia un receptor; los individuos interactuantes en la comunicación intercultural se adscriben a un conjunto de tradiciones, creencias, valores y forma de vida compartidas; finalmente, toda comunicación intercultural se sustenta en la negociación de significados compartidos para que el mensaje sea entendido. Además, la comunicación intercultural requiere de procesos de negociación de identidad donde ambos interactuantes tenderán a mantener y defender su autoimagen como miembro de un grupo determinado.

En consecuencia, un supuesto pedagógico importante de la presente propuesta es que una educación intercultural sustentada en el diálogo entre la cultura mapuche y la cultura no-mapuche que intercambian sus respectivos significados culturales con procesos de negociación de identidad cultural e imagen, apoyados por el profesor que redunden en identidad cultural y étnica positiva en los niños, en ambientes culturalmente familiares y contextos de relaciones cercanas significativas, y mediante el uso de estrategias didácticas que incorporen la diversidad étnicocultural a través del uso de la robótica educativa, permitirá al niño que se está formando experimentar seguridad y confianza en su identidad de membresía cultural, sentirse incluido, desear el contacto interpersonal, percibir estabilidad de su identidad en situaciones culturales predecibles, y adquirir nuevos conocimientos sobre la base de referentes culturalmente significativos.

Nueva redacción: En consecuencia, un supuesto pedagógico importante de la presente propuesta es que una educación intercultural debe sustentarse en el diálogo entre la cultura mapuche y la cultura no-mapuche. A través del diálogo y del apoyo del profesor, se intercambian los respectivos significados culturales con procesos de negociación de identidad cultural e imagen, cuyo objetivo es lograr una identidad cultural y étnica positiva en los niños. Mediante el uso de estrategias didácticas que incorporen la diversidad étnico-cultural, en ambientes culturalmente familiares y contextos de relaciones cercanas significativas, el uso de la robótica educativa permitirá al niño que se está formando experimentar seguridad y confianza en su identidad de membresía cultural, sentirse incluido, desear el contacto interpersonal, percibir estabilidad de su identidad en situaciones culturales predecibles, y adquirir nuevos conocimientos sobre la base de referentes culturalmente significativos. 


\subsection{ROBÓTICA EDUCATIVA E INTERCULTURAL}

En relación al uso de las TIC en educación, la informática educativa se ha integrado en el sistema educativo chileno desde principios de los años noventa (Hepp et al., 2004). Actualmente, el Programa Enlaces ha logrado que más del $96 \%$ de la matrícula escolar tenga acceso a las tecnologías digitales y más del $85 \%$ de los profesores de aula haya realizado cursos de capacitación para usos pedagógicos y administrativos de estas tecnologías. Hoy, la tasa de estudiantes por computador se sitúa en cerca de 13 y se pretende bajar a 10 en los próximos dos años. Más del $60 \%$ de los establecimientos educacionales cuenta con Internet y una proporción creciente de ellos, cercana al 50\%, dispone de banda ancha (Programa Enlaces, 2012). Junto al uso tradicional de computadores en las escuelas, algunos establecimientos educacionales han adquirido robots educativos, constituyendo talleres extra-programáticos con alumnos. A juzgar por las muestras organizadas por el programa Enlaces en diversas comunas del país, una cantidad creciente de escuelas y liceos se interesa por esta modalidad de uso de la informática.

En este contexto, los problemas que normalmente se les presentan a las escuelas respecto del uso pedagógico de las TIC, en particular en las escuelas subvencionadas, se pueden sistematizar en los siguientes ámbitos: costos de adquisición y mantención de equipos e Internet, información sobre el uso pedagógico para uso en el aula de las TIC en cada asignatura, e información general sobre el mercado proveedor de tecnologías de hardware y de software de mode de conocer y contrastar opciones de productos y servicios.

Sin embargo, a pesar de las investigaciones sobre el potencial educativo de las TIC (Hinostroza y Labé, 2011; Pedró, 2011; Hew y Brush, 2007), se han observado escasas propuestas que aborden el uso de estas tecnologías en las escuelas subvencionadas chilenas, y particularmente en el nivel de la Educación Parvularia o inicios de la Educación Básica con énfasis en la interculturalidad. Este proyecto pretendió llenar dicho vacío con una propuesta que integre un enfoque constructivista, el aspecto intercultural y una propuesta robótica simple y ajustada al currículum chileno, en especial en aquellas escuelas de mayor vulnerabilidad que buscan mejorar la efectividad de sus recursos.

La robótica educativa encuentra sus principales fundamentos en las teorías de aprendizaje construccionista. La teoría construccionista deviene del constructivismo y fue desarrollada por el equipo de Seymour Papert en el MIT (Papert y Harel, 1991). En el marco de esta teoría, se busca estimular el potencial creativo y expresivo a través de la comunicación de proyectos significativos, la resolución de problemas (estrategias de resolución) y el pensamiento lógico, el trabajo y el aprendizaje grupal en equipo, aprendiendo a respetar, negociar decisiones, desarrollar la autoestima, planificar tareas en conjunto y llevar a cabo el desarrollo de contenidos escolares específicos tales como la cuantificación, secuencias, orden, magnitudes, tamaños, figuras geométricas, trayectorias, motricidad fina y gruesa.

Para la operacionalización de las ideas planteadas anteriormente, juega un rol primordial el currículum educativo, pues permite concretar la teoría y guiar su puesta en marcha. Los Planes y Programas entregan un referente importante, en especial acerca de para qué y qué enseñar (Objetivos y Contenidos); pero es en torno al cómo hacerlo (didáctica) donde se manifiestan las principales dificultades. Nuestra propuesta pretende que la incorporación de la robótica no solo apunte al qué y para qué enseñar, sino más bien ofrece una mirada integral apoyando el cómo, a través de un material concreto y versátil. 
El uso de la robótica en ambientes interculturales resulta ser una interacción de variables poco estudiada hasta ahora, ya que las experiencias existentes en TIC e interculturalidad se relacionan principalmente con la creación y uso de material didáctico que se apoya en TIC, y cuya temática central se relaciona con la necesidad de preservar lenguas y dialectos, como también el conocimiento de las diferentes culturas (ver www.aulaintercultural.org).

Finalmente, para lograr efectividad con la robótica escolar en contextos escolares vulnerables, es necesario que profesores y estudiantes cuenten con materiales y herramientas que les permitan crear situaciones de aprendizaje a bajo costo, fácilmente adquiribles o que promuevan la reutilización de material disponible en sus entornos (hogar, barrio, campo). Otro aspecto importante es que estos materiales y herramientas deben ser atractivos, seguros y posibles de usar por estudiantes de todas las edades.

\subsection{EL PROYECTO}

El objetivo del proyecto de robótica intercultural fue abordar de manera interdisciplinar una propuesta educativa que propicie un diálogo intercultural a través de la robótica educativa e incorpore saberes y prácticas de la cultura mapuche y la cultura chilena permitiendo aprendizajes significativos en niños de segundo ciclo de Educación Parvularia y Primero Básico. Según el CENSO 2012, el 31.3\% de la población de La Araucanía se declaró como perteneciente a la etnia Mapuche $^{1}$. Las escuelas con que se trabajó en el proyecto son rurales, con alta proporción de estudiantes pertenecientes a la etnia Mapuche.

El proyecto propone la utilización de robots que pueden ser operados por cualquier niño, aunque no sepa leer (cualquier persona de 4 o más años capaz de pulsar teclas). Asimismo, el material didáctico tiene orientaciones de uso para profesores y apoderados. Para los profesores, los materiales incluyen guías impresas y digitales cuyos objetivos son: que profesores y apoderados conozcan el robot; que éste se utilice pedagógicamente con niños de acuerdo al currículum chileno (guías didácticas y fichas de trabajo); y que sea posible crear material didáctico de modo de adaptarlo a la realidad del aula y los estudiantes. El conjunto de robots y materiales didácticos que recibió cada escuela se denomina "Kit robótico". El robot -denominado "Cui Cui", que en Mapudungun significa "puente" - es un diseño electrónico, industrial y pedagógico de bajo costo, realizado íntegramente en Temuco en el marco de este proyecto.

\section{MÉTODO INVESTIGATIVO}

La investigación fue de tipo exploratorio y descriptivo; se utilizaron técnicas cualitativas y cuantitativas para identificar y describir experiencias que caracterizaron el uso del Kit en las escuelas observadas. En términos generales, se realizaron dos etapas en esta investigación, la primera de prueba de materiales, de instrumentos de evaluación y de prototipos de los robots. Esta etapa permitió organizar y plantear la segunda etapa de investigación de tipo cuasiexperimental.

Etapa 1: Prueba de materiales, instrumentos de evaluación y robots prototipo. Se seleccionaron 8 establecimientos educacionales de la Región de la Araucanía que mostraran

\footnotetext{
${ }^{1}$ Censo 2012, véase: Instituto Nacional de Estadísticas (INE) http://www.ine.cl/
} 
disposición para participar del pilotaje del proyecto. Los establecimientos educativos pertenecen a las comunas de Padre las Casas, Vilcún, Lautaro y Victoria. Se visitó cada escuela una vez por semana en horarios consensuados con cada docente dejando registro audiovisual de cada visita. En esta etapa se probaron los instrumentos de evaluación TEPSI, prueba de pre-cálculo, lista de cotejo, entrevista a profesores y encuesta con una escala de valoración Likert a los docentes, lo que permitió reformular los instrumentos y resguardar situaciones en la siguiente etapa.

Etapa 2: Investigación. Se realizó un estudio cuasi-experimental en 3 escuelas con grupo control y experimental en los niveles NT2 y $1^{\circ}$ Básico, urbano y rural de la comuna de Temuco. Se visitaron las escuelas experimentales dos veces por semana en horarios consensuados con cada docente, y se registró audiovisualmente cada visita.

Las técnicas cualitativas de investigación permitieron analizar la información en profundidad, buscando claves interpretativas para describir la situación en estudio y comprender las interacciones entre las distintas variables. Las técnicas cuantitativas permitieron describir atributos generales de los actores y de la realidad observada. Específicamente, en el diseño cualitativo se buscó describir la relación e interacción entre las variables de interés del estudio, las cuales comprenden: opinión de los profesores respecto del material y las dimensiones que se definieron a priori: 1) niño - robot (relación que establece el niño con el Robot); 2) niño soporte (relación que establece el niño con el soporte físico sobre el que se desplaza el Robot, este soporte contiene la unidad pedagógica específica a trabajar en cada sesión); 3) docente - Kit de robótica (relación entre el docente y el kit de robótica, el que se encuentra compuesto por la guía para el docente, recursos literarios, robot Cui Cui y fichas para el alumno); 4) docente - niño (relación entre el docente y el niño durante el uso del Kit); 5) interculturalidad.

La dimensión 5 de interculturalidad se refiere al conocimiento que los sujetos (docentes y alumnos) manejaban a nivel conceptual de la otra cultura, entendiendo que un primer paso para el respeto y la valoración es el conocimiento del otro de una manera más general, para luego avanzar a un nivel más profundo de interculturalidad, como lo es la aceptación, valoración y respeto. Por lo tanto, el análisis se centró en el nivel de conocimiento que los sujetos alcanzaron en el marco de las unidades didácticas trabajadas.

Las técnicas utilizadas fueron observación en aula con registro audiovisual para describir la relación interacción entre las variables mencionadas, y entrevista a los docentes para evaluar el kit de robótica. Las observaciones se complementaron con una lista de cotejo que registró el trabajo del docente y de los estudiantes con el Kit, la cual incorporó las dimensiones arriba descritas. Para finalizar el proceso de observación, se aplicó una entrevista y encuesta con una escala de valoración Likert a los docentes que implementaron la propuesta.

\section{PROCEDIMIENTO}

Luego de contactar a los directores de los establecimientos educacionales a los que se les solicitó su autorización y consentimiento para participar en la investigación, se hizo entrega del Kit de Robótica intercultural a los docentes, a quienes se capacitó en el uso de este material. Se les solicitó a los docentes destinar cierta cantidad de horas a la semana para el uso del material, esto quedó consensuado con la finalidad de visitar la utilización de esta herramienta tecnológica y dejar registro audiovisual de ello. 
Se les indicó a los docentes trabajar tres unidades didácticas asociadas al tema de salud, con énfasis en la integración de una visión desde lo mapuche como de la cultura occidental. Posteriormente, se realizó una entrevista semi estructurada a los docentes con el fin de evaluar el material entregado. Respecto de los resguardos éticos, éstos consideraron la autorización de los establecimientos educacionales, el resguardo de la integridad física y psicológica de los participantes, así como también mantener la confidencialidad de los informantes.

\section{4. $\quad$ ANÁLISIS DE LOS RESULTADOS}

Los datos se analizaron por medio del programa ATLAS Ti versión 6.2. Se utilizó análisis de contenido con categorías teóricas, en base a las cuales se estudiaron los datos de forma que permitieron describir las relaciones existentes entre las diversas dimensiones evaluadas. Los resultados se presentan en función de las dimensiones descritas y dan cuenta de factores que intervienen en el éxito en la implementación y apropiación del material. Éstos se resumen a continuación.

Dimensión "niño-robot Cui Cui": en el uso y manejo del Robot Cui Cui por parte de los niños, se observaron logros y dificultades. Dentro de los logros se destacan dos contenidos: el interés y entusiasmo con que los niños trabajaban con él y la rapidez con que los niños aprendieron el funcionamiento del robot. Respecto de las dificultades, éstas se relacionan con el rol del docente, principalmente en lo que se refiere a la organización y planificación de las actividades, ya que esto dificulta la calidad del trabajo y, por ende, la relación que establece el niño con el robot. Por último, respecto de la organización de la actividad se evidencia que cuando se trabaja con todo el curso existe distracción y pérdida de interés en la actividad por parte de los niños.

Dimensión "niño-soporte": esta dimensión busca facilitar el aprendizaje de los contenidos planificados en base a tres elementos. El primero es el material, es decir que el tamaño de las imágenes permita que al trabajar en grupo todos los niños sean capaces de observarlas, y que sea el desplazamiento del robot el que se adecúe al espacio físico, a la intención pedagógica y al contenido curricular. El segundo es la intención pedagógica y el tercero es el contenido curricular que trabaja la profesora.

Dimensión "Docente-Kit de robótica": esta dimensión hace referencia a la relación entre el docente y los diversos elementos del kit de robótica que se utilizan en diversos momentos de la planificación de la Unidad Didáctica. Por ejemplo, los recursos literarios se utilizan al comienzo de la planificación de las unidades didácticas. El robot y el soporte se utilizan durante el desarrollo de las unidades educativas para enseñar los contenidos de interculturalidad a los niños. A pesar de que este elemento parecía convocar la atención por lo innovador, no obtuvo los logros deseados. Durante el cierre de la unidad se utilizaron las fichas, ya que posibilitaron la reflexión sobre los aprendizajes con los niños.

Dimensión "Niño - Docente": durante el trabajo con el kit de robótica, se pudo observar que el docente puede asumir dos roles: asistencialista o facilitador. El docente asistencialista se caracteriza por prestar mucha atención al cuidado del robot y es muy directivo respecto del uso del mismo, lo que coarta e inhibe la interacción del niño con el recurso. Por el contrario, el docente facilitador provee las condiciones para que los niños puedan interactuar libremente con el robot. En cuanto al uso del Robot, éste se relaciona principalmente con el rol del docente. Por una 
parte, con el control del grupo y con ello la disciplina de los niños de manera que presten atención y se cumplan los objetivos de la actividad. Por otra, la preparación del contenido y las actividades a realizar, ya que en la medida que el docente lee el material complementario, sugerencias metodológicas, entre otros materiales proporcionados, es capaz de mediar más favorablemente el contenido intercultural en pos del aprendizaje del niño.

Dimensión "Interculturalidad": como se mencionó anteriormente, la interculturalidad comprende el conocimiento que alcanzan los niños respecto de la cultura mapuche. En general, en el trabajo de la propuesta pedagógica con el Kit robótico se observa que los niños logran con facilidad establecer paralelos entre la cultura occidental y la cultura mapuche, relatando historias populares que les han sido contadas en un contexto familiar y que dan cuenta de la representación social de los niños, ya que logran tipificar e identificar elementos propios de la cultura occidental y mapuche, por ejemplo, el kultrun, la Machi, el doctor, la farmacia, entre otros.

\subsection{ALGUNOS RESULTADOS RELEVANTES}

a) Compromiso del docente: el hecho de contar con los recursos en una sala no basta ni significa que los docentes los usarán de manera sistemática y adecuada. Cuando se evidencia compromiso y buena disposición al uso del Kit en el aula, se observa una mejor apropiación de parte de los docentes y alumnos en cuanto al uso y aplicación del material en actividades programadas.

b) Rol docente: el rol que asume el docente influye en el uso que se le da al material, es así como un docente con un rol más bien directivo, asistencialista o con dificultades para controlar su grupo no potencia una interacción provechosa entre los niños y el robot, sino más bien la limita y coarta. En cambio, aquellos docentes que asumen un rol mediador entre los niños y el recurso logran que sus alumnos trabajen en pequeños grupos de forma cooperativa y con mayor libertad para interactuar con el robot, hecho que posibilita una mejor apropiación del recurso por parte de los alumnos, en cuanto se propicia el uso y manejo autónomo del robot tanto en actividades libres como en aquellas planificadas. También se observó que los docentes no siempre leían la guía con las orientaciones para implementar las actividades, aspecto que conlleva a que la improvisación sea un elemento presente en muchas de las actividades asociadas a algún elemento del kit.

c) Organización del tiempo: en aquellos docentes en los que se observó una mejor organización, planificación y trabajo sistemático con el material asociado, se evidenció una mayor asimilación de los contenidos y uso del robot. En cambio, en aquellos docentes que trabajaron con el material de manera aislada y sin una organización clara de las actividades y tiempos para su ejecución, se observó un desaprovechamiento del material, menor apropiación del recurso como tal y de los contenidos. El factor tiempo fue una variable importante que determinó el uso con el material, así las docentes de Educación Parvularia mostraron mayor participación dado que la distribución del tiempo para ellas es más flexible, al contrario de lo que ocurrió en los niveles $1^{\circ}$ y $2^{\circ}$ Básico que están normados y presionados por una mayor carga horaria.

d) Actitud de los niños: en todas las ocasiones, se observó que los niños acogieron con mucho interés y curiosidad el robot, manifestando entusiasmo cada vez que trabajaron con él. Todos los niños, sin excepción, aprendieron y memorizaron con mucha facilidad el nombre del robot (cuicui, que significa puente en lengua mapuche). Los mejores resultados en cuanto a 
participación e interacción de los niños con el robot se observaron cuando el trabajo se ejecutó en pequeños grupos, lo que además posibilitó una mayor experticia en el uso del robot por parte de los niños.

\section{CONCLUSIONES}

La propuesta de este proyecto sienta un precedente en el uso de la robótica en los niveles iniciales de nuestro sistema educativo, el que además se encuentra asociado a la temática intercultural en el contexto de lo que significa vivir en la Región de la Araucanía, donde el 30\% del alumnado pertenece a la etnia mapuche. Sus resultados son auspiciosos en términos de la aceptación y apropiación por parte de los docentes del Kit pedagógico como un recurso didáctico, y de la positiva actitud de los alumnos en términos de su interés por trabajar con él.

Las actividades de inicio y cierre fueron las que tuvieron mejor aceptación por el docente, ya que estaban diseñadas desde una propuesta bastante tradicional, es decir, trabajo con todo el grupo o bien individual, donde el docente tiene el control de la actividad lo que le permite asumir un rol más bien directivo. Este hecho repercute en una mayor participación de los alumnos en función de la actividad que dirige el docente, aunque ello no fomenta al trabajo colaborativo, sino más bien se trata de una interacción micro-social de alumno-profesor.

Desde la escuela, este proceso se vio apoyado por las planificaciones y actividades contenidas en la guía del docente que forma parte del kit de robótica, en especial los recursos literarios que le permitieron a éste adentrarse en la temática de cada unidad didáctica a través de historias con las que los niños se sentían identificados y que permitieron introducir las actividades con el soporte y el robot Cui Cui.

Otro aspecto importante a destacar es el uso e internalización que los niños lograron de vocativos en mapudungun, comenzando por el nombre del robot hasta el uso de vocativos como lawen, ruka, kultrún, lawentuchefe, entre otros.

Los resultados más relevantes en el grupo experimental que usó la propuesta y los materiales en relación al grupo de control fueron: los alumnos de NT2 mostraron una tendencia positiva en el desarrollo del lenguaje; los alumnos de $1^{\circ}$ básico mostraron mayores logros en razonamiento lógico-matemático; se evidenció mayor conocimiento de la cultura mapuche por parte de los niños y de los docentes participantes; los docentes acogieron positivamente la propuesta como un recurso didáctico.

\section{REFERENCIAS BIBLIOGRÁFICAS}

Aula Intercultural, el Portal de la Educación Intercultural. http://www.aulaintercultural.org/

Bases curriculares de educación parvularia, 2005. Recuperado de

http://curriculumenlinea.mineduc.cl

Hepp, P., Hinostroza, J.E., Laval, E., y Rehbein, L. (2004). Technology in Schools: Education, ICT and the Knowledge Society. Washington: The World Bank. Recuperado de http://www.ceppe.cl/articulos-tecnologias-en-educacion/219-technology-in-schoolseducation-ict-and-the-knowledge-society-hinostroza-hepp-laval-rehbein

Hew, K. y Brush, T., (2007). Integrating Technology into K-12 teaching and learning: Current 
knowledge gaps and recommendations for future research. Educational Technology Research and Development, $n$. 55, 223-252.

Hinostroza, J. y Labbé, C. (2011). Políticas y prácticas de informática educativa en América Latina y el Caribe. Santiago: CEPAL. División de Desarrollo Social. Serie Políticas Sociales $\mathrm{N}^{\circ} 171$.

Merino, M., Mellor, D., Quilaqueo, D. y Saiz, J. L. (2009). Perceived discrimination amongst the indigenous Mapuche people in Chile: Some comparisons with Australia. Ethnic and Racial Studies, vol.32, n.5, 802-822.

Merino, M., Quilaqueo, D. y Saiz, J. (2008). Tipología del discurso de la discriminación percibida en Mapuches de Chile. Revista Signos, n. 41, 67, 279-297.

MINEDUC (2005). Bases Curriculares de Educación Parvularia. Recuperado de http://curriculumenlinea.mineduc.cl

Política de interculturalidad en educación parvularia. Documento oficial del Ministerio de Educación de Chile. http://www.mineduc.cl/index2.php?id seccion=3043\&id portal=16\&id contenido=12131

Papert, S. y Harel, I. (1991). Situating constructionism. En http://www.papert.org/articles/SituatingConstructionism.html

Pedró, F. (2011). Tecnología y escuela: lo que funciona y por qué. Documento Básico. XXVI Semana Monográfica de la Educación LA EDUCACIÓN EN LA SOCIEDAD DIGITAL. Recuperado http://www.fundacionsantillana.com/upload/ficheros/noticias/201111/documento bsico.pdf

Programa Enlaces (2012), Ministerio de Educación. Síntesis de resultados Censo TIC 2012. Recuperado de http://www.enlaces.cl/index.php?t=44\&i=2\&cc=1679\&tm=2

Schmelkes, S. (2006). La interculturalidad en la educación básica. Ponencia presentada en la Segunda Reunión del Comité Intergubernamental del Proyecto Regional de Educación para América Latina y el Caribe (PRELAC), Santiago de Chile, 11 al 13 de mayo de 2006.

SIMCE (2012). Resultados prueba Simce año 2012. Recuperado de http://www.simce.cl

Ting-Toomey, S. (1999). Communicating across cultures. New York: The Guilford Press. 